\title{
Klinische Pharmazie trifft klinische Pharmakologie
}

\author{
Barbara Lardi-Studler \\ Dr. sc. nat., freischaffende, journalistisch tätige Apothekerin
}

Die wachsenden Anforderungen, z.B. durch immer komplexere Therapien, spezielle Patientenpopulationen, Zunahme an chronischen Patienten, Polymorbidität und nicht zuletzt auch Schwierigkeiten bei der Medikamentenbeschaffung, stellen in der medikamentösen Therapie eine grosse Herausforderung an die Spezialisten dar. Die klinische Pharmazie will den sicheren, rationalen und ökonomisch sinnvollen Gebrauch von Heilmitteln entwickeln und fördern (Definition der European Society of Clinical Pharmacy ESCP); diese sehr allgemein gefassten Ziele verfolgen letztlich auch klinische Pharmakologen. Umso wichtiger ist der Dialog zwischen der klinischen Pharmazie und der klinischen Pharmakologie, damit Synergien genutzt, Redundanzen vermieden und wo möglich Arbeitsabläufe vereinfacht werden können. Nur so kann eine bestmögliche medikamentöse Versorgung der Patienten sichergestellt werden.

\section{Klinische Pharmazeuten und klinische Pharmakologen im Spital}

(Aus den Referaten von Dr. phil. Markus Lampert, Prof. Dr. med. Dr. pharm. Stephan Krähenbühl, Prof. Dr. Chantal Csajka, Prof. Dr. med. Thierry Buclin, Delia Bornand, eidg. dipl. Apothekerin FPH Spitalpharmazie)

Die Begriffe klinische Pharmazie und klinische Pharmakologie verwirren nicht nur die Patienten - sogar bei Medizinalpersonen führt dies immer wieder zu Missverständnissen. Pharmakokinetik und Pharmakodynamik sind zwar integrale Bestandteile der pharmazeutischen Wissenschaften, im klinischen Alltag grösserer Spitäler beschäftigen sich aber vorwiegend die Pharmakologen damit. Umso wichtiger ist es daher, die einerseits verschiedenen und andererseits aber doch ähnlichen Tätigkeitsbereiche zu definieren, letztlich auch, um die begrenzten personellen Ressourcen optimal zu nutzen. Bei durchschnittlich etwa $10 \%$ aller Patienten treten Adverse Drug Events (ADE) auf, und ungefähr ebenso viele Arzneimittelanwendungen beinhalten Fehler, so dass es mehr als genug zu tun gibt für beide Berufssparten.
Je nach Spitalgrösse wird die Situation sehr unterschiedlich erlebt. Arbeitet ein klinischer Pharmakologe im Spital, ist er in viel engerem Kontakt mit dem Patienten selber, während der klinische Pharmazeut mehr um die Sicherheitsaspekte der Arzneimitteltherapie bemüht ist. In einer universitären Einrichtung arbeiten klinische Pharmazeuten und klinische Pharmakologen heute überwiegend ergänzend zusammen. In kleineren Spitälern übernehmen aber oft die klinischen Pharmazeuten zwangsläufig auch Aufgaben der klinischen Pharmakologen, weil das Spital über keine Klinik oder Abteilung für klinische Pharmakologie verfügt.

Der klinische Pharmazeut kennt den Medikationsprozess als Ganzes; er versteht die arzneimittelbezogenen Risiken sowohl auf individueller Ebene (patientenspezifische Risiken, Interaktionen, UAW usw.) als auch auf systemischer Ebene (Prozesse und Abläufe im Spital). Als akademische Medizinalperson mit der Weiterbildung zum Fähigkeitsausweis FPH in klinischer Pharmazie bringt er dafür das nötige Rüstzeug mit.

Durch gezielte Interventionen kann der klinische Pharmazeut zu einer Verbesserung in der Sicherheit des Medikationsprozesses und der Arzneimitteltherapie beitragen. Die Erfahrungen zeigen, dass je nach Klinik in bis zu 85\% der Fälle die Empfehlungen der klinischen Pharmazeuten auf der Visite umgesetzt werden. In einigen Fällen der Arzneimitteltherapie ist ein tiefer gehendes Verständnis der Klinik notwendig, und da kommt der klinische Pharmakologe zum Zug.

Die Kernziele seiner Weiterbildung richten sich neben den Grundlagen u.a. auf die Erfassung des individuellen Nutzen-Risikos für den Patienten, Arzneimittelstudien, individualisierte Therapie und Erfassung der Sicherheit und Ökonomie der Arzneimitteltherapie. Insbesondere bei komplexen Fragestellungen (z.B. Therapie bei Organversagen), dem Management von Interaktionen und unerwünschten Arzneimittelwirkungen, speziell bei polymorbiden Patienten, oder der Interpretation von therapeutischem Monitoring, wäre ein Konzil des klinischen Pharmakologen wünschenswert. Relevante Beobachtungen sollen unbedingt auch 
publiziert werden. Als Resultat solcher Interventionen ist am CHUV beispielsweise eine Webseite entstanden, auf der Dosisanpassungen bei kontinuierlicher Nierenersatztherapie berechnet werden können (continuous renal replacement therapy; www.crrt.ch). Damit wird auch ersichtlich, dass klinische Pharmakologen neben ihrer klinischen auch auf akademische Tätigkeit (Lehre und Forschung) ausgerichtete Ärzte sind. Deshalb arbeiten sie momentan überwiegend in Universitätsspitälern, wo sie diese Aufgaben wahrnehmen können. Oftmals sind die Patienten jedoch nicht genügend lange im Primärspital, als dass sie mit dem klinischen Pharmakologen über ihre Medikation sprechen könnten. In dieser Lücke liegt ein grosses Potential für die klinischen Pharmazeuten - nicht nur im Spital, sondern auch in der Offizin.

\section{Klinische Pharmazeuten in der Offizin}

(Referat von Prof. Dr. Olivier Bugnon)

Der klinisch tätige Apotheker kann eine Reihe von Dienstleistungen erbringen, die für das Gesundheitswesen von grossem Nutzen sind, wie z.B.

- Medikationsanalysen

- Adhärenzkonsultationen

- Coaching von Patienten z.B. mit chronischen Erkrankungen, inkl. HIV

- Individualisierte Therapie speziell bei vulnerablen Gruppen

- Risikomanagement

- Austausch von Problemsituationen und Arbeitsabläufen mit den Ärzten (Qualitätszirkel)

Ein grosses Feld für die ambulante klinische Pharmazie ist die pharmazeutische Nachbetreuung von Spitalpatienten nach dem Spitalaustritt. Oftmals verstreichen 1-2 Wochen, ehe der Patient seinen Hausarzt konsultiert. In dieser Zeit ist er meist sich selber überlassen, hat zu Hause noch die alten Medikamente, versteht u.U. nicht, welche er jetzt nehmen muss usw. In dieser kritischen Phase kann der Apotheker Klarheit schaffen. Bei Prof. Bugnon in der Apotheke des PMU in Lausanne (Policlinique médicale universitaire) werden daher oft auch Hausbesuche durchgeführt, bei denen

Weiterbildung Fähigkeitsausweis FPH in klinischer Pharmazie Voraussetzung: Grundlagen der Pharmakologie im Bachelorund Master-Studiengang;

Dauer: mind. 1 Jahr nach dem Master-Studiengang bei 100\% Anstellung, überwiegend jedoch teilzeitlich über mehrere Jahre (Anpassung des Weiterbildungsganges ist im Gange).

Zahl der Absolventen: 5-10 pro Jahr;

Weitere Informationen bei der GSASA:

www.gsasa.ch $\rightarrow$ Bildung man die Hausapotheke nach Spitalaustritt sortiert und die Therapie bespricht und erklärt. Damit wurden sehr gute Erfahrungen gemacht und die Dienstleistung wurde auch von den Hausärzten sehr geschätzt. Diese Zusammenarbeit nach dem Spitalaustritt wird zurzeit in Lausanne mit Ärzten, Krankenschwestern und Pharmazeuten gemeinsam diskutiert.

\section{Ökonomische Aspekte}

(Referat von Prof. Dr. Christoph Meier)

Ökonomische Aspekte werden heute immer relevanter. Wenn man von einer Inzidenz von 10\% für Adverse Drug Events (ADE) ausgeht und pro ADE zusätzliche Kosten von 5000 CHF annimmt, ergibt dies pro Bett und Jahr 19000 CHF, also für ein Spital der Grösse des Basler Universitätsspitals 12,5 Mio. CHF potentielle Mehrkosten pro Jahr - eine stolze Summe, welche die Arbeit von klinischen Pharmazeuten und Pharmakologen wohl mehr als rechtfertigen würde. Allerdings bleibt es schwierig, die effektiven Einsparungen durch klinische Pharmazeuten zu zeigen. Dessen ungeachtet darf man nicht vergessen, dass klinische Pharmazeuten den Fokus auf einer Station verändern und einen Schulungseffekt auf Pflege und Ärzteschaft haben, welcher sich ebenfalls in einer besseren Pharmakotherapie niederschlägt und sich mehrfach multipliziert. Auch für die Offizin sind Apotheker mit einer Ausbildung in klinischer Pharmazie wichtige Multiplikatoren.

\section{Zusammenfassung}

Beide Berufe bauen auf den Grundlagen der Pharmakologie auf. Diese Grundkenntnisse erlauben Tätigkeiten, die von beiden Berufen auf ihre Art und Weise ausgeübt werden können. Dazu gehören insbesondere die Erfassung und Gewährleistung der Sicherheit des Patienten allgemein (Dosierungsprüfung, UAW, Interaktionen, Mehrfachverschreibungen) und individuell (z.B. bei vulnerablen Gruppen wie geriatrischen $\mathrm{Pa}$ tienten, Schwangeren und Kindern) sowie auch in Vertretung der Gesellschaft (bei Behörden). Diese Tätigkeiten sind für den klinischen Pharmazeuten auch in der Offizin möglich.

Neben diesen Parallelen gibt es Gebiete, die klar dem einen oder anderen Berufsstand unterliegen, weil dieser jeweils die dafür besten Voraussetzungen mitbringt. Klinische Pharmakologen sind Ärzte und verbinden bei der Anwendung der Arzneimitteltherapie die pharmakologischen Kenntnisse mit dem gesamten klinischen Kontext des Patienten, insbesondere mit den Diagnosen und den durchgeführten Untersuchungen. Klinische Pharmazeuten sind Apotheker und fo- 


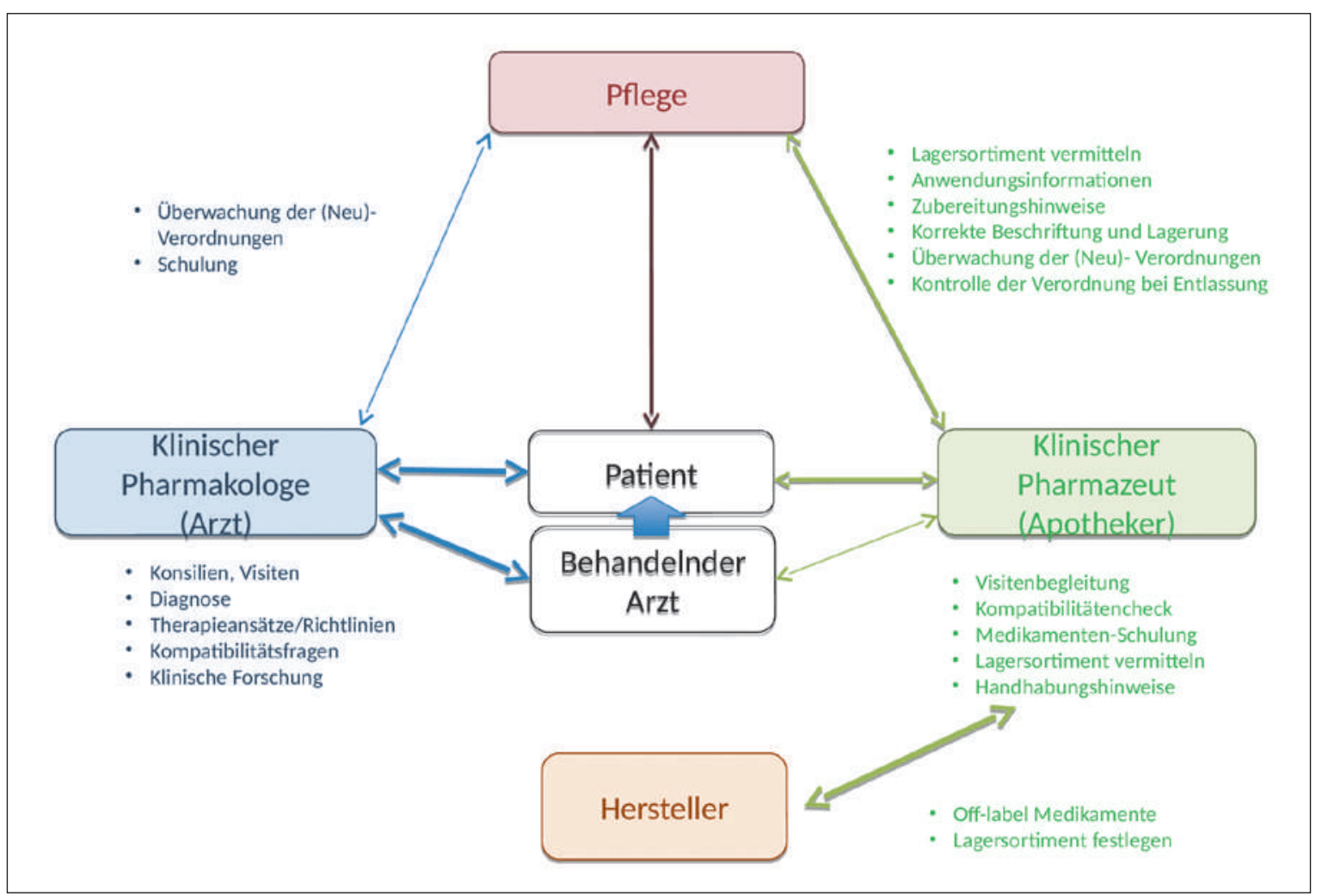

Abbildung 1: Der optimale Benefit für den Patienten kommt durch eine gute interdisziplinäre Zusammenarbeit zustande. Dargestellt ist eine Auswahl möglicher interdisziplinärer Interaktionen zwischen beiden Berufsgruppen.

kussieren neben der Pharmakologie auf die rein alltäglichen und praktischen Aspekte der Arzneimitteltherapie (Herstellung, Bereitstellen des richtigen Medikamentes in der richtigen Form) mit Blick auf die optimale Wirksamkeit und Sicherheit in der Handhabung des Arzneimittels oder die Förderung der Compliance im gesamten Patientenkollektiv.

In vielen Spitälern im Ausland sind es die klinischen Pharmazeuten, welche die Arzneimitteltherapie sowohl verschreiben als auch überwachen. In der Schweiz fliesst zum jetzigen Zeitpunkt die Expertise beider Spezialisten ein. Dieses «Miteinander» ist ein riesiger

Weiterbildung Facharzttitel Klinischer Pharmakologe und Toxikologe, FMH

Voraussetzung: Grundlagen der Pharmakologie im Bachelorund Masterstudium;

Dauer: Weiterbildung mindestens 6 Jahre (je 3 Jahre Innere Medizin, Pädiatrie oder Anästhesie und fachspezifische Weiterbildung). Die meisten Absolventen haben 5 Jahre Weiterbildung Innere Medizin absolviert, bevor sie die Weiterbildung Klinische Pharmakologie antreten. Dies ist wichtig, weil die meisten Anfragen die Innere Medizin betreffen und klinische Pharmakologen direkt in den Medikationsprozess eingreifen können.

Zahl der Absolventen: 4-6 pro Jahr;

Weitere Informationen bei der SGKPT:

www.clinpharm.ch/Pharmaco/ $\rightarrow$ Weiterbildung
Vorteil unseres Gesundheitssystems, den wir uns immer wieder vor Augen führen sollen und müssen. Die beiden Berufspartner brauchen einander und ergänzen sich gemeinsam, um zu einem optimalen Benefit für den Patienten beizutragen (Abb. 1).

Es ist wünschenswert, dass möglichst viele junge Kollegen in beiden Berufsständen die Weiterbildung absolvieren. Dabei wären gemeinsame Module in der Aus- und Fortbildung wünschenswert, um die interdisziplinäre Kommunikation zu fördern und das unterschiedliche Denken zu verstehen und zu nutzen. Wenn wir momentan noch am «Verhandeln» sind, was beide Berufsgruppen zu tun und zu lassen haben, so wird der Ausbau bei den Spezialisten in beiden Berufsgruppen dafür sorgen, dass sich der vollständige Austausch und das Miteinander ergeben werden. Die SAPhW versteht es als notwendige Chance, die beiden Berufe auch im Konsens mit der SAMW näher zusammenzubringen und die Synergien zu nutzen. Als Akademie hat sie die notwendige fachliche und wissenschaftliche Berechtigung und ist beiden Berufen gegenüber offen. Dies ist für eine professionell betreute Arzneimitteltherapie von grossem Patientennutzen und spricht letztlich auch für ein gutes pharmakoökonomisches Ergebnis.

Gekürzte Fassung des Vorabrucks im pharmaJournal 8/15 (mit Genehmigung von pharmaSuisse). 